\title{
Existence of a Solution to the Discrete Theodorsen Equation for Conformal Mappings
}

\author{
By Martin H. Gutknecht*
}

\begin{abstract}
The discrete Theodorsen equation is the basis of efficient numerical methods for conformal mappings of the unit disk onto Jordan regions that are starlike with respect to the origin. Applying Brouwer's fixed point theorem we show here that there always exists a solution to this equation.
\end{abstract}

Let $D$ denote the unit disk (in the $z$-plane) and $G$ a region (in the $w$-plane) with boundary $\Gamma$, where $\Gamma$ is a Jordan curve that is starlike with respect to the origin and thus defined by polar coordinates $\tau, \rho(\tau), \rho$ being a $2 \pi$-periodic positive continuous function. (Note that 'starlike' sometimes is defined slightly more general.) Then there exists a unique mapping function $f: \bar{D} \rightarrow \bar{G}$ that is continuous in $\bar{D}$, conformal in $D$, and normalized by $f(0)=0, f^{\prime}(0)>0 . f$ is already determined by a real $2 \pi$ periodic function $\theta$ satisfying $f\left(e^{i \phi}\right)=\rho[\theta(\phi)] e^{i \theta(\phi)}$. For one of these functions Theodorsen's integral equation holds [2, p. 65]:

$$
\theta(\phi)=\phi+\frac{1}{2 \pi} \mathrm{P} \cdot \mathrm{V} \cdot \int_{0}^{2 \pi} \log \rho[\theta(\sigma)] \cot \frac{\phi-\sigma}{2} d \sigma .
$$

The existence of $f$ implies that this nonlinear singular integral equation always has at least one strictly monotone continuous solution. One can show [2, Theorem 1.3 , p. 66] that there exists only one solution with these properties. Moreover, if $\Gamma$ satisfies a so-called $\epsilon$-condition with $\epsilon<1$, then (1) cannot have additional nonmonotone continuous solutions.

Definition. A starlike Jordan curve defined by polar coordinates $\tau, \rho(\tau)$ satisfies an $\epsilon$-condition if $\rho$ is absolutely continuous and

$$
\epsilon:=\operatorname{ess}_{0 \leqslant \tau \leqslant 2 \pi}\left|\frac{\rho^{\prime}(\tau)}{\rho(\tau)}\right|<\infty .
$$

For numerical computations Eq. (1) needs to be discretized. The easiest and most useful way to do this [2] , [3] leads to what we call the discrete Theodorsen equation:

$$
\theta=\phi+\mathbf{K} \log \rho(\theta)
$$

Here $\phi, \theta, \log \rho(\theta)$ are $n$-dimensional vectors ( $n$ even) with components $\phi_{k}:=$ $2 k \pi / n, \theta_{k}$, and $\log \rho\left(\theta_{k}\right), k=1, \ldots, n$, respectively, and $\mathrm{K}$ is Wittich's matrix:

Received July 8, 1976.

AMS (MOS) subject classifications (1970). Primary 30A28; Secondary 65R05.

Key words and phrases. Discrete Theodorsen equation, Theodorsen's integral equation, numerical conformal mapping, Brouwer's fixed point theorem.

* This work was supported by NRC (Canada) Grant \#A8240. 
(4)

$$
(\mathbf{K})_{k j}:=\left\{\begin{array}{l}
0, \text { if } j-k \text { even, } \\
\frac{2}{n} \cot \frac{(k-j) \pi}{n}, \quad \text { if } j-k \text { odd, }
\end{array} \quad(k, j=1, \ldots, n) .\right.
$$

Of course, $\theta_{k}$ is supposed to approximate $\theta\left(\phi_{k}\right)$.

Again, it can be shown [2, Theorem 3.2, p. 87] that Eq. (3) has exactly one solution if $\Gamma$ satisfies an $\epsilon$-condition with $\epsilon<1$. Moreover, in this case the solution can be computed by direct iteration or by variants of Newton's method. These numerical methods are very efficient even for large $n$ (i.e. fine discretization) because the fast Fourier transform can be applied [3]. In addition, for a quite general class of boundaries $\Gamma$, which must be symmetric about an axis but may have an arbitrary large $\epsilon$, the local convergence of the iteration method modified by underrelaxation is established in [3] supposing the existence of a solution. Surprisingly, it is possible to prove this existence assuming only the continuity of $\rho$.

THEOREM. If $\rho$ is continuous, the discrete Theodorsen equation (3) has a solution.

Proof. We define $r: \bar{D} \rightarrow \mathbf{R}^{+}$by

$$
r(z):=\left\{\begin{array}{l}
\rho(\operatorname{Arg} z)|z|+1-|z|, \quad \text { if } 0<|z| \leqslant 1 \\
1, \quad \text { if } z=0 .
\end{array}\right.
$$

(Arg denotes the principal value of the argument, $-\pi<\operatorname{Arg} z \leqslant \pi$. But since $\rho$ is $2 \pi$-periodic, we could use an arbitrary branch.) The function $r$ is continuous. Using the notation $r(\mathrm{z}):=\left(r\left(z_{1}\right), \ldots, r\left(z_{n}\right)\right)^{T}, \exp (\mathrm{w})=\left(\exp \left(w_{1}\right), \ldots, \exp \left(w_{n}\right)\right)^{T}$, etc., we define $\Psi: \bar{D}^{n} \rightarrow(\partial D)^{n}$ by

$$
\Psi(\mathrm{z}):=\exp [i \phi+i \mathrm{~K} \log r(\mathrm{z})] .
$$

$\boldsymbol{\Psi}$ is a continuous function mapping a convex compact subset of $\mathbf{C}^{\boldsymbol{n}}$ into itself.

According to Brouwer's fixed point theorem $\left[1\right.$, p. 454], $\Psi$ has a fixed point $z^{*} \in$ $\Psi\left(\bar{D}^{n}\right) \subset(\partial D)^{n}$. But for $z \in \partial D$ we simply have $r(z)=\rho(\operatorname{Arg} z)$; thus

$$
\begin{aligned}
\mathbf{z}^{*} & =\exp \left[i \phi+i \mathbf{K} \log \rho\left(\operatorname{Arg} \mathbf{z}^{*}\right)\right], \\
\operatorname{Arg} \mathbf{z}^{*} & =\phi+\mathbf{K} \log \rho\left(\operatorname{Arg} \mathbf{z}^{*}\right)-2 \pi \mathbf{l}
\end{aligned}
$$

for some integer vector 1 . So, we set $\theta^{*}:=\operatorname{Arg} z^{*}+2 \pi l$ implying

$$
\theta^{*}=\phi+\mathbf{K} \log \rho\left(\theta^{*}\right)
$$

i.e. $\theta^{*}$ is a solution to $(3)$.** $^{* *}$

**Note Added in Proof. Another easy proof can be based on Schauder's more powerful second fixed point theorem [1, p. 456] (or an analogic theorem for finite-dimensional spaces) instead of Brouwer's theorem. 
Remarks. (i) If $\Gamma$ is symmetric about an axis (or about several axes), then one can similarly prove that there exists a solution with corresponding symmetry. Of course, one has to assume that the point $\operatorname{set}\left\{\exp i \phi_{k} \mid k=1, \ldots, n\right\}$ is also symmetric about the axis.

(ii) Examples in [3] show that solutions to (3) are not necessarily monotone.

Department of Computer Science

University of British Columbia

Vancouver, B.C. V6T 1W5, Canada

1. L. COLLATZ, Functional Analysis and Numerical Mathematics, Springer-Verlag, Berlin and New York, 1964; English transl., Academic Press, New York, 1966. MR 29 \#2931; 34 \#4961.

2. D. GAIER, Konstruktive Methoden der konformen Abbildung, Springer-Verlag, Berlin and New York, 1964. MR 33 \#7507.

3. M. H. GUTKNECHT, "Solving Theodorsen's integral equation for conformal maps with the fast Fourier transform. I," Numer. Math. (To appear.) 